disturbances, including difficulty in finding words, substitution of one word for another with the same meaning, and slowing down of verbal expression. No such effects were reported in the two control groups.

On neuropsychiatric testing of language function (performed in the no-headache period), all patients in the topiramate group scored lower than those in the other two groups; the patients on topiramate with language disturbances had the highest (worst) scores for all test variables. The presence of psychiatric comorbidities, especially anxiety and depression, resulted in worse results for some language functions.

The neuropsychological test scores were higher (worse) than normal values in all tests, for all patient groups. The authors hypothesize that all patients with migraine could have cognitive (and particularly language) disturbances, as the pathways involved in migraine pain processing could facilitate language disturbances as an expression of a more generalized impairment of cognitive processing. Topiramate could, however, further the impairment of cognitive functioning. The authors recommend additional prospective studies to investigate these issues.

Original article Coppola F et al. (2007) Language disturbances as a side effect of prophylactic treatment of migraine. Headache [doi: 10.1111/j.1526-4610.2007.00860.x]

\section{Primary somatosensory cortical involvement in idiopathic RLS}

The involvement in idiopathic restless legs syndrome (RLS) of brain regions associated with central somatosensory processing has been suggested, but has not been confirmed by high-resolution MRI studies of the whole brain. Previous studies have indicated the involvement of basal ganglia, thalamus, infratentorial structures and spinal cord in this condition. Unrath et al. have used a voxel-based morphometry approach to test the hypothesis that patients with RLS exhibit volume changes in sensorimotor and pain processing areas of the cortex, and in the thalamus.

Analysis of 63 patients with idiopathic RLS (mean disease duration 22.3 years) and 40 healthy controls identified regional decreases in gray matter volume that were significantly associated with RLS. Regional decreases were located bihemispherically in the primary somatosensory cortex; local volume decrease was more widespread in the left hemisphere (cluster size 2,563 vs 1,363 voxels for left side vs right side). Left hemispheric changes were also observed in primary motor areas (precentral gyrus). Gray matter volume at the centroids of the three significant clusters correlated positively with duration of disease, and negatively with patients' scores on the International Restless Legs Severity Scale. No local increases in gray matter volume were observed in RLS patients compared with controls.

The authors conclude that their study provides the first in vivo evidence of involvement of the somatosensory cortex in idiopathic RLS. Their data will contribute to the ongoing task of elucidating the pathophysiology of the condition.

Original article Unrath A et al. (2007) Cortical grey matter alterations in idiopathic restless legs syndrome: an optimized voxel-based morphometry study. Mov Disord

[doi: $10.1002 / \mathrm{mds} .21608$ ]

\section{Virus-derived peptide enables transvascular delivery of small interfering RNA to the CNS}

Gene silencing by RNA interference is a promising therapeutic approach for neurological disorders, but the blood-brain barrier presents an obstacle to safe and efficient delivery of interfering RNA to neurons - a problem that has, until now, only been circumvented by direct intraparenchymal injection. Kumar et al. hypothesized that this obstacle could be overcome by exploiting the ability of neurotropic viruses to cross the blood-brain barrier, and these authors have now shown that intravenous injection of antiviral small interfering RNA (siRNA) bound to a short virus-derived peptide offers protection against fatal viral encephalitis in mice.

The authors demonstrated that a synthetic chimeric peptide (RVG-9R) consisting of a 29-residue peptide derived from rabies virus glycoprotein (RVG) and an additional nine arginine residues could deliver siRNA specifically to mouse neuronal cells in vitro and in vivo, resulting in effective gene silencing. Flow cytometry analysis following intravenous injection of mice with a complex of fluorescent siRNA and RVG-9R revealed that the siRNA was taken up in the brain but not the liver or spleen, indicating that transvascular RVG-9R 